\title{
Impact of cancer rehabilitation on the prognosis of patients with hepatocellular carcinoma
}

\author{
RYUKI HASHIDA ${ }^{1,2}$, TAKUMI KAWAGUCHI ${ }^{3}$, SHUNJI KOYA ${ }^{1}$, KEISUKE HIROTA ${ }^{1}$, NORIHIRO GOSHIMA ${ }^{4}$, \\ TERUHITO YOSHIYAMA ${ }^{4}$, TAKASHI OTSUKA ${ }^{4}$, MASAFUMI BEKKI ${ }^{1,2}$, SOHEI IWANAGA $^{1,2}$, DAN NAKANO $^{3}$, \\ TAKASHI NIIZEKI ${ }^{3}$, HIROO MATSUSE ${ }^{1,2}$, ATSUSHI KAWAGUCHI $^{5}$, NAOTO SHIBA $^{2}$ and TAKUJI TORIMURA ${ }^{3}$ \\ ${ }^{1}$ Division of Rehabilitation, Kurume University Hospital; ${ }^{2}$ Department of Orthopedics, Kurume University \\ School of Medicine; ${ }^{3}$ Division of Gastroenterology, Department of Medicine, Kurume University School of Medicine; \\ ${ }^{4}$ Department of Medicine, Kurume University School of Medicine, Kurume, Fukuoka 830-0011; \\ ${ }^{5}$ Center for Comprehensive Community Medicine Faculty of Medicine, Saga University, Saga, Saga 849-8501, Japan
}

Received November 12, 2019; Accepted November 25, 2019

DOI: $10.3892 / \mathrm{ol} .2020 .11345$

\begin{abstract}
Sarcopenia is a prognostic factor for patients with hepatocellular carcinoma (HCC). Cancer rehabilitation (CR) improves patients' physical function and muscle mass. We investigated the effects of $\mathrm{CR}$ on the prognosis of patients with HCC. The present study was a prospective observational study, which analyzed 152 patients with HCC who underwent transcatheter arterial chemoembolization (TACE) between 2013 and 2016. Patients were classified into the $C R(n=85)$ and control $(n=67)$ groups. The effects of $C R$ on muscle mass were evaluated by changes in the skeletal muscle index (SMI) before and after TACE. Independent factors associated with survival were evaluated by Cox regression analysis. Kaplan-Meier analysis was used to compare the survival rate between the CR and control groups. The difference in survival rate between the two groups was also examined after propensity score matching. SMI was significantly increased in the CR group compared with the control group. In Cox regression analysis, independent factors associated with survival were CR and Child-Pugh class A (estimate 1.760, 95\% CI 0.914-3.226, $\mathrm{P}=0.001$; estimate $1.602,95 \%$ CI 0.426-2.998, $\mathrm{P}=0.0129$ ). The survival rate was significantly higher in the CR group than in the control group (median 552 vs. 424 days; $\mathrm{P}=0.0359$ ). The survival rate was also significantly higher in
\end{abstract}

Correspondence to: Dr Takumi Kawaguchi, Division of Gastroenterology, Department of Medicine, Kurume University School of Medicine, 67 Asahi-machi, Kurume, Fukuoka 830-0011, Japan

E-mail: takumi@med.kurume-u.ac.jp

Abbreviations: BCAA, branched-chain amino acid; CLD, chronic liver disease; CR, cancer rehabilitation; CT, computed tomography; HCC, hepatocellular carcinoma; SMI, skeletal muscle index; TACE, transcatheter arterial chemoembolization; VFA, visceral fat area

Key words: sarcopenia, hepatoma, SMI, survival, exercise the CR group than that in the control group after propensity score matching (median 529 vs. 369 days; $\mathrm{P}=0.0332$ ). $\mathrm{CR}$ was associated with prolonged survival in patients with HCC who underwent TACE. Patients with cancer are recommended to maintain physical activity even during cancer treatment.

\section{Introduction}

Exercise is recommended for patients with various diseases including cancer $(1,2)$. Walking as well as resistance training has been reported to improve physical function and skeletal muscle mass in patients with cancer $(3,4)$. In accordance with the American College of Sports Medicine guidelines, exercise training is a key recommendation to maintain activity even in cancer patients (5). Cancer rehabilitation (CR), a new multidisciplinary intervention for cancer patients, consists of nutritional and physical therapy. CR improves fatigue, pain, physical function, and quality of life in patients with cancer (6).

Sarcopenia is defined as loss of skeletal muscle mass and function. Sarcopenia frequently occurs in patients with chronic liver disease (CLD) regardless of its etiology $(7,8)$. Sarcopenia in patients with CLD is associated with a decline in their quality of life and physical activity (9). Sarcopenia is also significantly associated with hepatic fibrosis (10). Moreover, sarcopenia is an independent prognostic factor for hepatocellular carcinoma (HCC) (11). Taken together, it is important to pay attention to sarcopenia in patients with CLD.

In patients with cirrhosis and esophageal varices, moderate-intensity exercise increases portal pressure and may, therefore, increase the risk of variceal bleeding (12). In addition, moderate-intensity exercise stimulates the renal vasoconstrictor system, markedly impairing the renal function in patients with cirrhosis and ascites (13). In this way, exercise may exacerbate the general condition of patients of cirrhosis with complications such as esophageal varices and ascites. However, Hiraoka et al (14) showed that branched-chain amino acid (BCAA) supplementation and low-intensity exercise are effective for improving muscle volume and strength in patients with CLD. In addition, Locklear et al (15) demonstrated that, even in patients with end-stage liver disease, 
exercise has potential benefits in terms of endurance and functional outcome measures without adverse effects. We also previously reported that $\mathrm{CR}$ improved not only physical ability but also muscle mass without worsening liver function during hospitalization for cancer treatment in patients with CLD and advanced HCC $(16,17)$.

Thus, CR has beneficial effects on sarcopenia in patients with CLD and HCC; however, the effects of CR on prognosis remain unclear in patients with CLD and advanced HCC. This study aimed to investigate the effects of $\mathrm{CR}$ on the prognosis of patients with CLD and advanced HCC.

\section{Patients and methods}

Study design. CR for in-hospital patients with cancer is an approved health care service covered by health insurance from the Ministry of Health, Labour and Welfare in Japan. Therefore, intentionally using a non-exercise control group is contrary to medical ethics. Thus, we performed a prospective cohort study to evaluate effects of CR on skeletal muscle mass during hospitalization for patients with HCC and CLD who underwent TACE.

Ethics. The study was conducted in accordance with the ethical guidelines of the Declaration of Helsinki, as reflected in the prior approval given by the institutional review board of Kurume University (approval no. 15072). Informed consent was obtained from an opt-out approach, and personal information was protected during data collection.

Subjects. A total of 152 consecutive patients with HCC and CLD from February 2013 to October 2016 participated in this study. Inclusion criteria were hospitalized patients with HCC and CLD who (1) were 20 years of age or older, (2) had a performance status of grade 0 to 2 as defined by the Eastern Cooperative Oncology Group (18), and (3) had undergone treatment with TACE. Exclusion criteria were patients with (1) risk of HCC rupture, (2) history or presence of grade 2-4 hepatic encephalopathy according to the West Haven Criteria (19), (3) risk of esophageal or gastric varices rupture, (4) heart failure, or (5) respiratory failure. The exclusion criteria were assessed upon study enrollment. All patients underwent upper gastrointestinal endoscopy by gastroenterologists every 6 to 12 months, and the risk of esophageal or gastric varices rupture was assessed according to the 2015 evidence-based clinical practice guidelines for liver cirrhosis from the Japanese Society of Gastroenterology (20). The definition of heart failure was based on guidelines from the American College of Cardiology Foundation/American Heart Association Task Force and Heart Failure Society of America $(21,22)$. The definition of respiratory failure was based on guidelines from the European Respiratory Society/American Thoracic Society $(23,24)$. Outpatient doctors and nurses recommended exercise to all patients. Patients who agreed to exercise were classified into the CR group ( $\mathrm{n}=85)$, and those who did not agree to exercise were classified into the control group ( $\mathrm{n}=67)$. We classified enrolled patients into the High SMI or Low SMI group according to the JSH criteria for sarcopenia (7), and the High VFA or Low VFA group as previously described (25). We also divided the patients into the High or Low LDH group and the High total protein group or Low total protein group based on the reference values of serum LDH and total protein levels, respectively.

Exercise regimen. To maintain physical ability and prevent sarcopenia, patients in the CR group were treated with exercise, instructed by physical therapists certified in the rehabilitation of cancer patients. The rehabilitation was started on the day following TACE unless the patient had a fever of $38^{\circ} \mathrm{C}$ or greater as previously described (16). The frequency and duration of exercise performed were recorded during the hospitalization. After discharge, patients in the CR group were instructed to continue the exercises by themselves during the observation period.

According to the guidelines of American College of Sports Medicine (26), the exercise consisted of the following 4 types of training (median 2.5 metabolic equivalents $/ 20-40 \mathrm{~min} / \mathrm{day}$ ): 1) stretching, 2) strength training, 3) balance training, and 4) endurance training $(16,17)$. A static stretching was conducted for 3-5 min, targeting the quadriceps femoris muscles, hamstrings, hip adductor muscles, and gastrocnemius. The static stretch was held to each muscle for 10-20 seconds at the point of feeling tightness $(16,25)$. Strength training was conducted for $10 \mathrm{~min}$, targeting the quadriceps femoris muscles, gastrocnemius, and tibialis anterior muscles. Quadriceps femoris muscle and iliopsoas strength training were conducted at moderate to high intensity by a hand-held dynamometer. Gastrocnemius and tibialis anterior muscles were conducted trained by the patient's weight. One set contained of 10 repetitions, and a maximum of 3 sets were performed (16,25). Balance training contain the patients practicing a one-leg stance and tandem stance for 5-10 min. The patient stood on a straight line and maintain posture in tandem stance training $(16,25)$. Endurance training contained either ergometer or walking for 10-15 min. The intensity of endurance training was 11-13 points on the Borg scale, and the target heart rate was set by using the Karvonen Formula $(16,25)$.

Diagnosis, the Barcelona Clinic Liver Cancer (BCLC) staging system, tumor node metastasis (TNM) staging, and treatment of HCC. HCC was diagnosed by a combination of tests for serum tumor makers, such as alpha-fetoprotein and des- $\gamma$-carboxy prothrombin, and imaging procedures, such as ultrasonography, computed tomography (CT), magnetic resonance imaging (MRI), and angiography, or a tumor biopsy (27). We evaluated the clinical stage of $\mathrm{HCC}$ using the Barcelona Clinic Liver Cancer (BCLC) staging system (27) and TNM staging based on the Liver Cancer Study Group of Japan criteria (28). We treated the patients according to clinical practice guidelines for HCC of The Japan Society of Hepatology (29).

Additional treatment for recurrence of HCC after initial TACE. When HCC recurred, additional treatment for $\mathrm{HCC}$ was selected based on The Japan Society of Hepatology Consensus-Based Treatment Algorithm for HCC (28). Moreover, additional HCC treatment with hepatic arterial infusion chemotherapy/tyrosine kinase inhibitors was selected when HCC showed TACE failure/refractoriness defined by any of following criteria: i) an ineffective response after two or more consecutive TACE procedures that is evident on response evaluation CT or MRI 
after 1-3 months, even after chemotherapeutic agents are changed and/or the feeding artery is reanalyzed; ii) two or more consecutive progressions in the liver (including an increase in the number of tumors compared to that before the previous TACE procedure), even after changing the chemotherapeutic agents and/or reanalysis of feeding artery, on response evaluation CT/MRI after 1-3 months following adequately performed selective TACE; iii) continuous elevation of tumor markers right after TACE even though transient minor reduction is observed; iv) appearance of vascular invasion, or v) appearance of extrahepatic spread (28).

Measurement of skeletal muscle index (SMI) and visceral fat area (VFA). SMI and VFA were evaluated using CT images obtained before and after TACE. The first CT images were obtained to evaluate HCC before TACE. The second CT images were obtained to evaluate the effectiveness of TACE against HCC. VFA was measure at slices at the umbilical level (29). We measured skeletal muscle mass and VFA by using manual tracings on CT images, and their sum was calculated using Image-J software (National Institutes of Health, Bethesda, MD, USA) (30). Skeletal muscle mass was normalized by the square of the height, and the data was expressed as SMI.

Measurement of physical function. Grip strength, 10-meter walking speed, and 6-min walking test were evaluated by qualified physical therapists. Handgrip was measured on the non-dominant hand using a dynamometer (TKK5401; Takei Scientific Instruments Co., Ltd.) according to Japan Society of Hepatology guidelines (7). The 10-meter walking speed was measured to evaluate gait speed. To evaluate the 10-meter maximal gait time, 2 meters were added to allow for acceleration before and deceleration after the 10-meter gait test, respectively (31). The 6-min walking test was measured by evaluating the total ambulated distance according to the American Thoracic Society guidelines (32).

Biochemical tests. We measured following blood biochemical tests; aspartate aminotransferase, alanine aminotransferase, alkaline phosphatase, gamma-glutamyl transpeptidase, albumin, total bilirubin, creatinine, estimated glomerular filtration rate, creatine kinase, hemoglobin Alc, prothrombin activity, and platelet count. We also measured serum levels of $\alpha$-fetoprotein, des- $\gamma$-carboxy prothrombin.

Changes in variables before and after TACE. Changes in SMI and VFA between pre- and post-TACE were evaluated by the $\triangle \mathrm{SMI}$, and $\triangle \mathrm{VFA}$. In addition, $\triangle \mathrm{SMI}$ and $\triangle \mathrm{VFA}$ were evaluated by a stratification analysis according to sex. Similarly, changes in each physical function were evaluated by the $\Delta$ variables. All $\Delta$ variables were evaluated by change in the variable $(\Delta$ variable $=$ variable after TACE-variable before TACE)

Definition of event and follow-up. An event was defined as death from any cause in this study. After discharge, patients were followed up until death or the study censor date by routine physical examinations, biochemical tests, and ultrasonography, CT, or magnetic resonance imaging according to the HCC guidelines of the Japan Society of Hepatology (29). The median observation period was 511 days (range, 13-1180 days).
Statistical analysis. Data are expressed as the median (interquartile range $[\mathrm{IQR}]$ ), range, or number. The differences between the control and CR groups were analyzed by using Wilcoxon rank sum tests. The difference $\triangle \mathrm{SMI}$ in $\mathrm{CR}$ group among the etiologies of liver disease (multiple groups) was examined by analysis of variance followed by Scheffe's post hoc test. The difference $\Delta$ SMI between female and male or Child-Pugh class $\mathrm{A}$ and $\mathrm{B} / \mathrm{C}$ were analyzed by using Wilcoxon rank sum tests. The level of statistical significance was set at $\mathrm{P}<0.05$.

A multivariate Cox regression analysis with a stepwise variable selection was used to identify independent variables associated with the prognosis of patients with HCC. In the present study, we did not conduct a univariate analysis, and the explanatory variables were selected in a stepwise manner, minimizing the Bayesian information criterion as previously described (33). Overall survival analysis was performed using the Kaplan-Meier method followed by the log-rank test.

Propensity scores for all the patients were estimated by a logistic regression model using the following baseline characteristics as covariates: Age, sex, BCLC stage, Child-Pugh score, and BCAA supplementation as previously described (2). To generate 88 pairs of patients, we employed a one-to-one nearest-neighbor matching algorithm with an optimal caliper of 0.2 without replacement. The c-statistic in this study was 0.603, and 88 CLD patients with HCC (control $[n=44]$ and CR $[\mathrm{n}=44])$ were analyzed.

All the statistical analyses were performed using $\mathrm{JMP}^{\circledR} 13$ software (SAS Institute Inc.) or the R software package ( $R$ Foundation for Statistical Computing, 2012).

\section{Results}

Patient characteristics. Patient characteristics data were obtained before TACE. The characteristics, the CR and control group are shown in Table I. Patients in the CR group were significantly older than those in the control group. The VFA in the CR group was significantly higher than that in the control group. However, there was no significant difference in the female-to-male ratio, body mass index, or SMI between the $\mathrm{CR}$ and control groups. No significant difference was seen in TNM stage, BCLC classification, or levels of $\alpha$-fetoprotein and des- $\gamma$-carboxy prothrombin. There was no significant difference in Child-Pugh classification, hemoglobin A1c value, or estimated glomerular filtration rate between the $\mathrm{CR}$ and control groups (Table I).

No significant difference was observed between the CR and control groups regarding the prevalence of patients treated with BCAA supplementation, hospitalization period, or the evaluation interval for CT (Table I). There was no significant difference in the number of TACE sessions at the baseline, number of TACE sessions during the observation period, or additional $\mathrm{HCC}$ treatment with hepatic arterial infusion of chemotherapy/tyrosine kinase inhibitors during the observation period (Table I). There were no patients who underwent liver transplants during the observation periods.

During the hospitalization, the median length of therapeutic exercise was 6.5 days (IQR, 5-9 days; range, 2-26 days) in the CR group with a median of $3.33 \mathrm{~h}$ of exercise performed (IQR, 2.67-4.42 h; range, 1-8.67 h). 


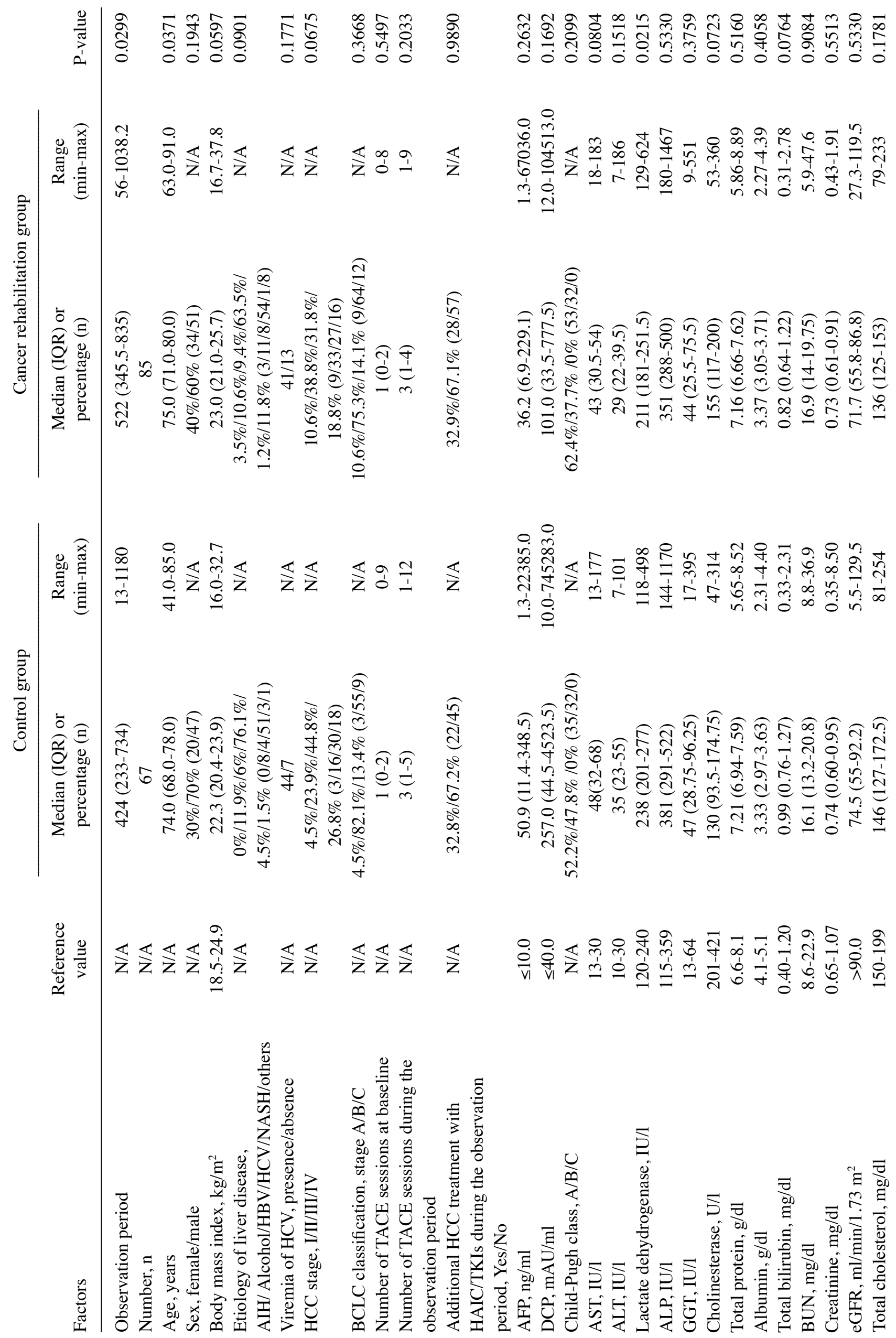




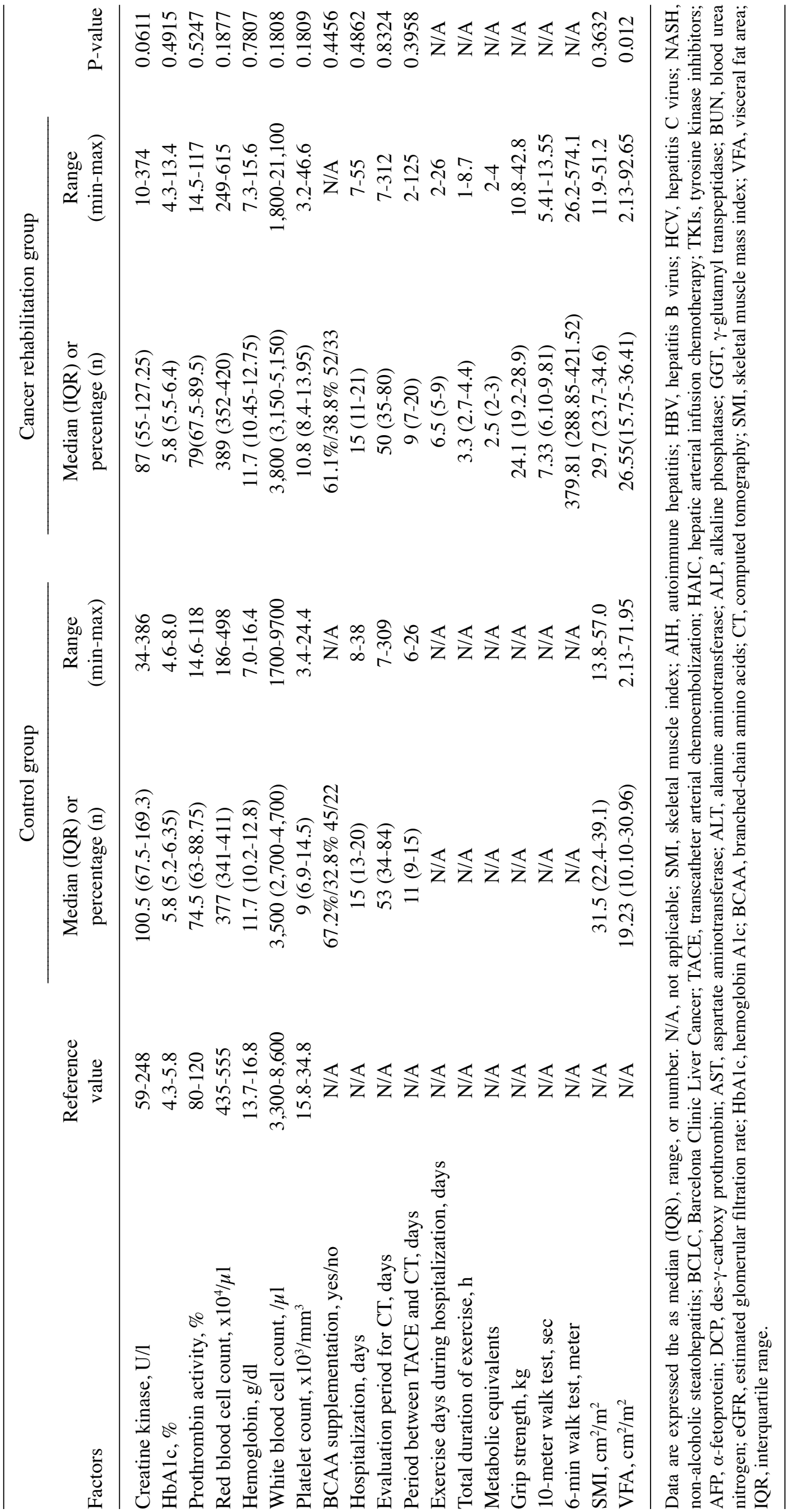



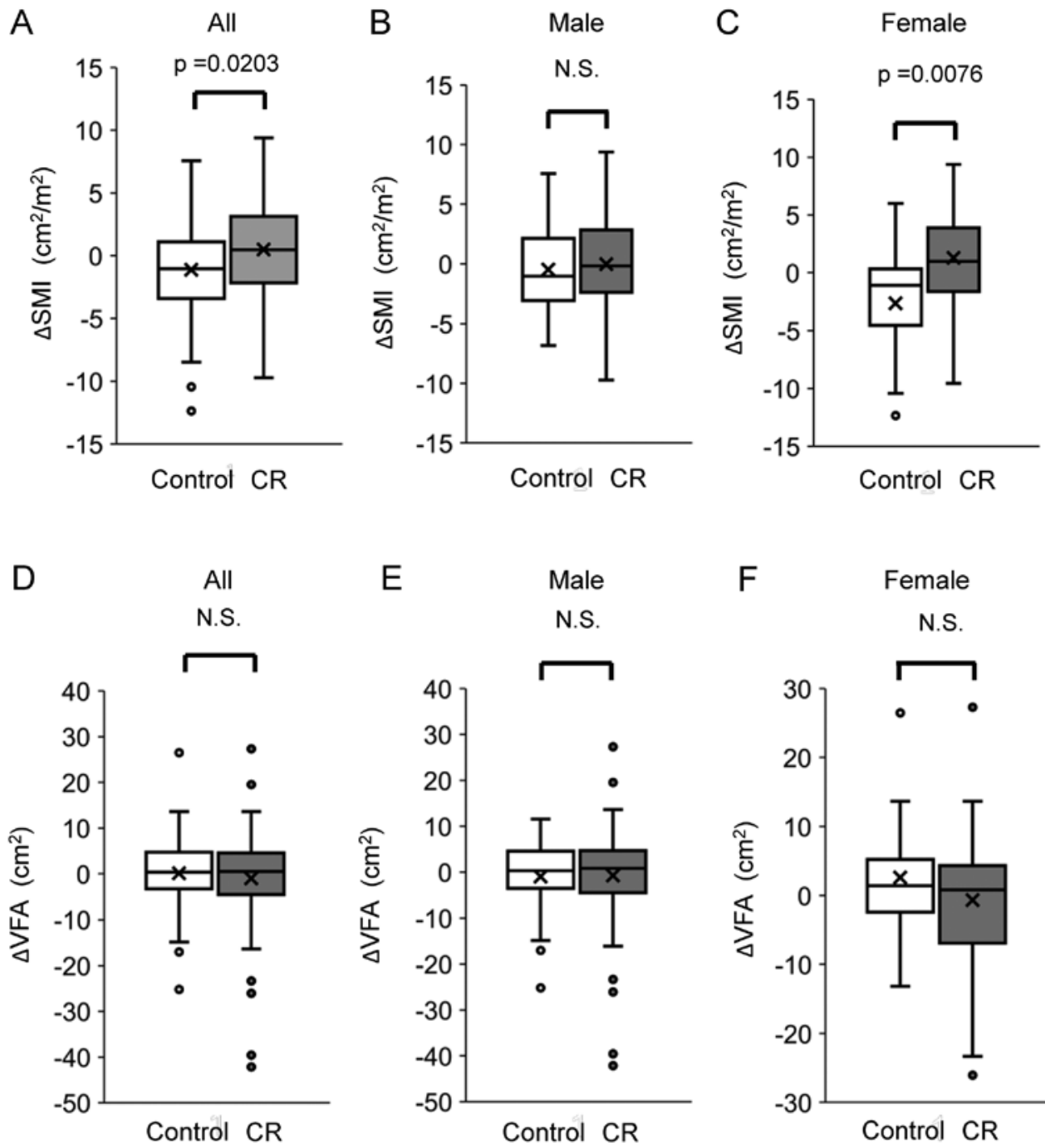

E

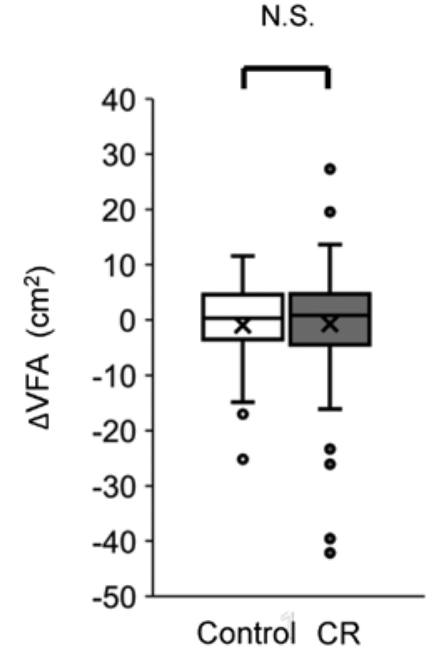

$\mathrm{F}$

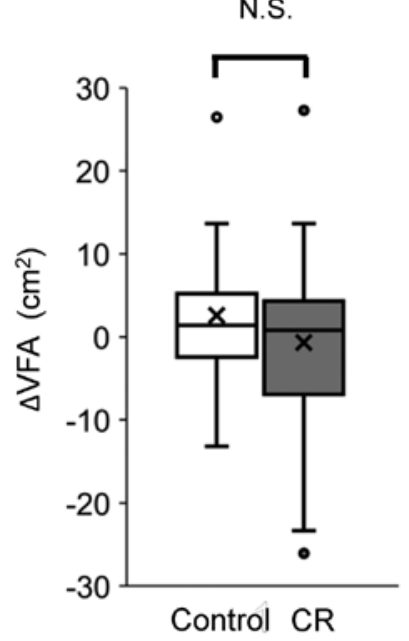

Figure 1. Difference in $\triangle \mathrm{SMI}$ between the CR and control groups in (A) all subjects, and (B) male and (C) female patients. Difference in $\triangle \mathrm{VFA}$ between the $\mathrm{CR}$ and control groups in (D) all subjects, and (E) male and (F) female patients. CR, cancer rehabilitation; SMI, skeletal muscle index; VFA, visceral fat area; N.S., not significant.

Changes in SMI and VFA. We evaluated changes in SMI by the difference in SMI between before and after TACE. After treatment with TACE, $\triangle \mathrm{SMI}$ was significantly higher in the $\mathrm{CR}$ group than in the control group. There was no significant difference between the $\mathrm{CR}$ and control groups regarding the $\triangle \mathrm{SMI}$ in male patients. On the other hand, there was a significant difference between the $\mathrm{CR}$ and control groups regarding $\Delta$ SMI in female patients (Fig. 1). The $\Delta$ SMI had no significant association with the etiology of liver disease in the CR group (Fig. S1). Moreover, in the CR group, $\triangle$ SMI was not significantly different between male and female patients or between patients classified as Child-Pugh class A and Child-Pugh class B/C (Fig. S1B and C). There was no significant difference in the $\triangle \mathrm{VFA}$ between the $\mathrm{CR}$ and control groups. Moreover, there was no significant difference in VFA between the CR and control groups in both male and female patients (Fig. 1).

Correlations between survival period and $\Delta$ each variable. Correlations between survival period and the alterations in body composition and physical performance were evaluated using pairwise correlations. There was no significant correlation between the survival period and $\triangle \mathrm{SMI}$ or $\triangle \mathrm{VFA}$ (Table II). Moreover, no significant correlation was seen between the survival period and $\Delta$ grip strength, $\Delta 10$ meter walking speed, or $\Delta 6$-min walking test (Table II).

Associations between survival period and changes in SMI, and baseline SMI, VFA, and serum levels of $L D H$ and total protein. We performed stratification analysis according the changes in SMI. There was no significant difference in survival rate between the patients with increased and decreased SMI in all patients (median 526 vs. 459 days, $\mathrm{P}=0.6206$; Fig. 2). The impact of changes in SMI on survival was also examined in the CR and control groups. No significant difference was seen in survival between the patients with increased SMI and decreased SMI in both the CR and control groups (CR group; increased SMI vs. decreased SMI; median 541 vs. 569 days, $\mathrm{P}=0.3856$; control group: Increased SMI vs. decreased SMI, median 379 vs. 432 days, $\mathrm{P}=0.5201$; Figs. S2A and B). We also performed stratified analysis for survival according to the several baseline clinical parameters. There was no significant difference in survival rate between the High and Low SMI 
Table II. Pairwise correlations between survival period and $\Delta$ each variable.

\begin{tabular}{lcc}
\hline Variables & Correlation coefficient & P-value \\
\hline$\Delta$ SMI & -0.00029 & 0.9971 \\
$\Delta$ VFA & -0.03869 & 0.6338 \\
$\Delta$ grip strength & -0.09365 & 0.4767 \\
$\Delta 10$ m walking speed & -0.04024 & 0.9121 \\
$\Delta 6$-min walk test & -0.19864 & 0.1712
\end{tabular}

SMI, skeletal muscle mass index; VFA, visceral fat area.

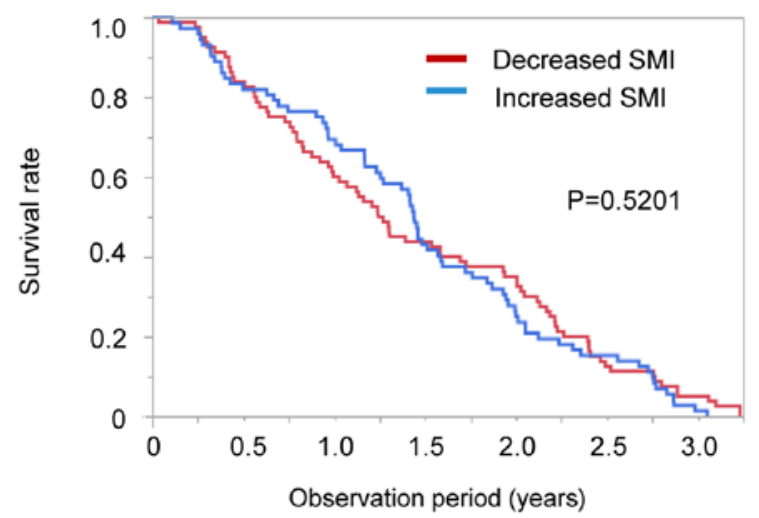

Patient number at risk

$\begin{array}{cccccccc}\text { Increased SMI } & 47 & 42 & 37 & 23 & 13 & 10 & 1 \\ \text { Decreased SMI } & 80 & 67 & 49 & 35 & 28 & 10 & 3\end{array}$

Figure 2. Difference in survival rate between the groups with increased and decreased SMI. SMI, skeletal muscle index.

groups $(\mathrm{P}=0.7064)$ and between the High and Low VFA groups $(\mathrm{P}=0.7530)$. There was no significant difference in the survival rate between the High and Low LDH groups $(\mathrm{P}=0.1095)$. There was no significant difference in the survival rate between the High and Low total protein groups $(\mathrm{P}=1.0330)$.

Independent factors associated with survival. We evaluated the independent factors associated with survival using Cox regression analysis. There was significant difference in serum level of LDH and total protein between the CR and control groups in univariate analysis. However, these factors were not identified as an independent factor for survival in the Cox regression analysis. The $\mathrm{CR}$ group and Child-Pugh class $\mathrm{A}$ were identified as independent positive factors associated with survival (Table III). Although BCLC stage was not significantly associated with survival, the number of TACE sessions at baseline were an independent negative factor for survival (Table III). The number of TACE sessions during the observation period and additional HCC treatment with hepatic arterial infusion of chemotherapy/tyrosine kinase inhibitors during the observation period were not selected by a stepwise procedure.

We also evaluated independent factors associated with survival in the CR and control groups respectively. In the CR group, the number of TACE sessions during the observation and Child-Pugh class were identified as independent factors
Table III. Independent factor for survival.

\begin{tabular}{|c|c|c|c|}
\hline Factors & Estimate & $\begin{array}{l}95 \% \text { Confidence } \\
\text { interval }\end{array}$ & P-value \\
\hline Group (CR) & 1.760 & $0.914-3.226$ & 0.0010 \\
\hline $\begin{array}{l}\text { Child-Pugh } \\
\text { (class A-B) }\end{array}$ & 1.602 & $0.426-2.998$ & 0.0129 \\
\hline $\begin{array}{l}\text { Number of TACE } \\
\text { session at baseline }\end{array}$ & -0.843 & $-1.465-0.337$ & 0.003 \\
\hline
\end{tabular}

N/A, not applicable; CR, cancer rehabilitation; TACE, transcatheter arterial chemoembolization.

for survival ( $\mathrm{P}=0.0004$ and $\mathrm{P}=0.0278)$. In the Control group, BCLC stage was identified as an independent factor for the survival $(\mathrm{P}=0.0034)$.

Impact of CR on survival period. Kaplan-Meier analysis was used to compare the survival rate between the CR and control groups. The median observation period was 511 days (range, 13-1180 days). The survival rate of the $\mathrm{CR}$ group was significantly higher than that of the control group on Kaplan-Meier analysis (median 552 vs. 424 days, $\mathrm{P}=0.0359$; Fig. $3 \mathrm{~A}$ ).

Impact of $C R$ on survival period after propensity score matching. After propensity score matching, we evaluated overall survival by Kaplan-Meier analysis. Propensity scores for all the patients were estimated using the following baseline characteristics as covariates: Age, sex, BCLC stage, Child-Pugh score, and BCAA supplementation. There was no significant difference in observation period between the CR and control groups (Table IV). No significant difference was seen in number of TACE session at baseline and SMI between the two groups (Table IV). The survival rate of the CR group was significantly higher than that of the control group (median 529 vs. 369 days, $\mathrm{P}=0.0332$; Fig. $3 \mathrm{~B}$ ).

\section{Discussion}

In this study, we investigated effects of $\mathrm{CR}$ on the prognosis of patients with HCC who underwent TACE. The survival rate was higher in HCC patients with CR than in HCC patients without CR. Cox regression analysis demonstrated that $\mathrm{CR}$ was an independent factor for survival. These results may indicate that $\mathrm{CR}$ has beneficial effect on prognosis of patients with HCC who underwent TACE.

In the present study, SMI significantly increased in the CR group without worsening liver function in patients with CLD and HCC who underwent TACE. Dawson et al (34) showed that $\mathrm{CR}$ improves muscle mass in patients with prostate cancer during androgen treatment. We also reported previously that $\mathrm{CR}$ increased muscle mass in patients with HCC during treatment (14). Taken together, CR may improve the muscle mass of patients with cancer in spite of treatment for cancer.

In the female, but not in the male, $\Delta \mathrm{SMI}$ in the CR group was significantly higher than that in the control group. This gender difference could be accounted for a marked decrease 


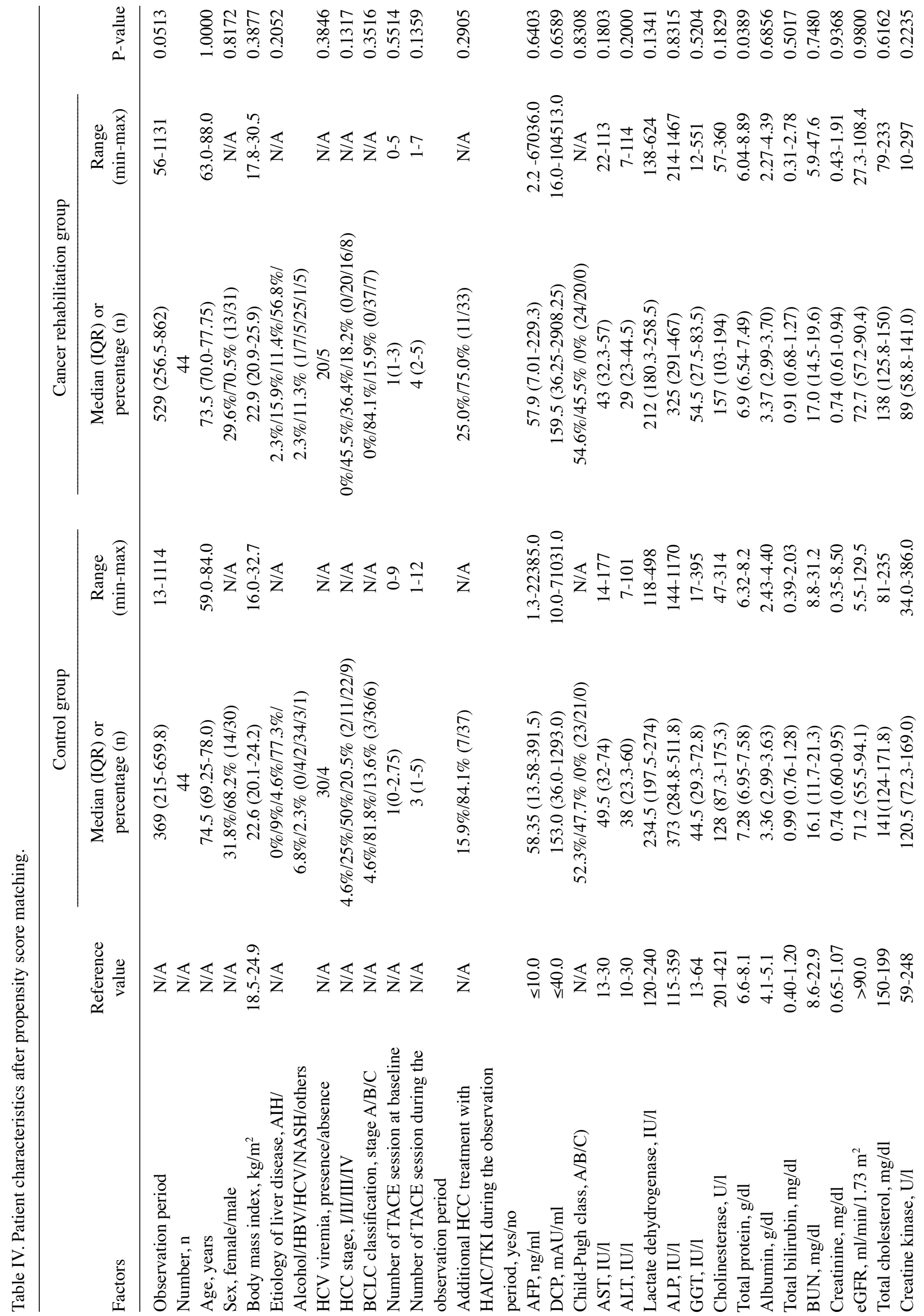




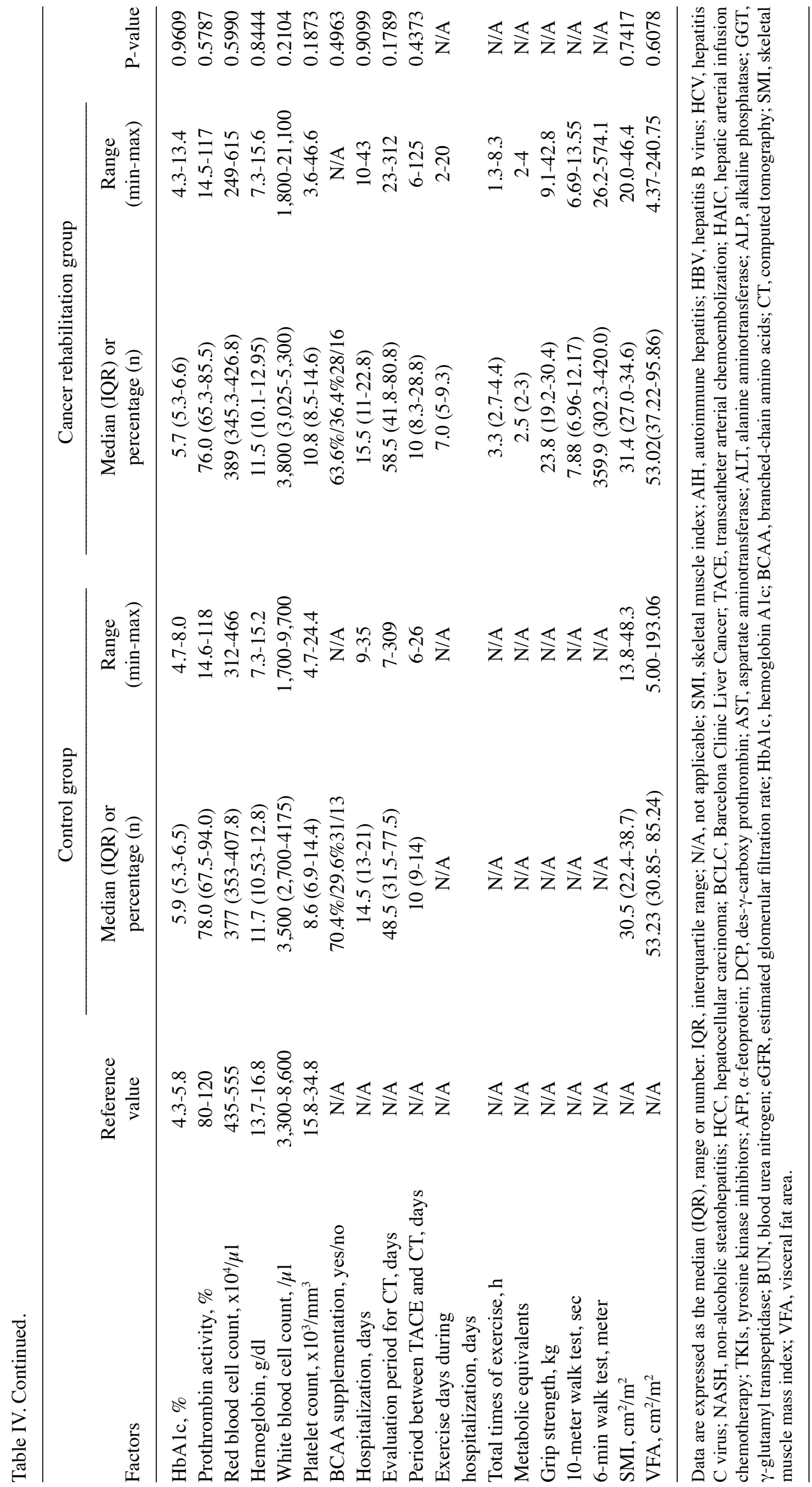


A

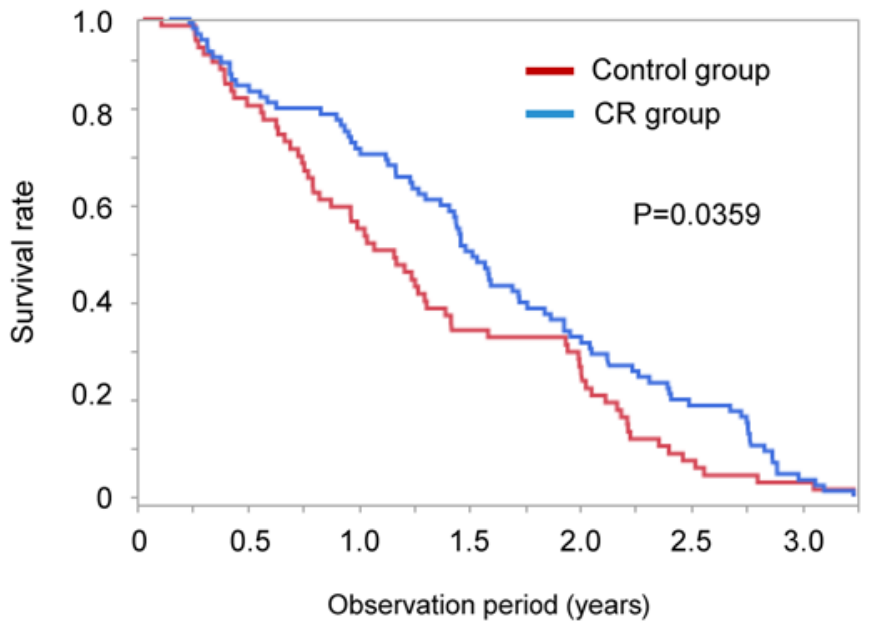

Patient number at risk

$\begin{array}{rrrrrrrr}\text { Control } & 67 & 55 & 38 & 23 & 18 & 5 & 2 \\ \text { CR } & 85 & 72 & 61 & 43 & 28 & 17 & 3\end{array}$

B

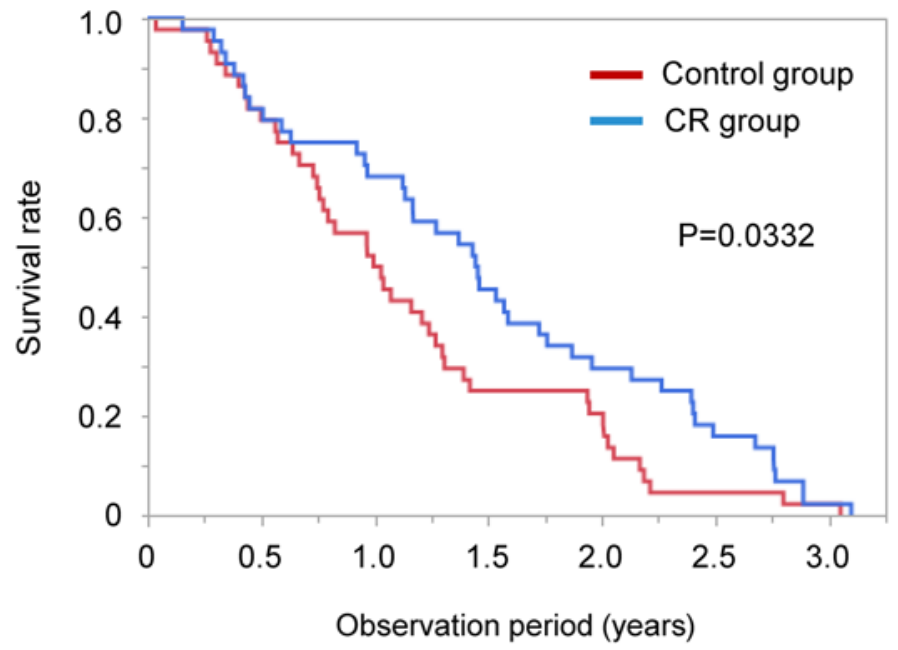

Patient number at risk

$\begin{array}{rlllllll}\text { Control } & 44 & 36 & 23 & 12 & 10 & 3 & 1 \\ \text { CR } & 44 & 37 & 31 & 21 & 14 & 7 & 1\end{array}$

Figure 3. (A) Difference in survival rate between the CR and control groups. (B) Difference in survival rate between the CR and control groups after propensity score matching. CR, cancer rehabilitation.

of $\Delta \mathrm{SMI}$ in female of the control group. In our previous study, skeletal muscle mass was also significantly decreased in female than in male HCC patients who underwent TACE (35). A possible reason for the gender difference is sex hormones. Testosterone has potent anabolic effects on skeletal muscle, leading to muscle protein synthesis and subsequently increases muscle mass (36). Women have low serum testosterone levels and serum free testosterone levels are reported to be positively correlated with skeletal muscle mass in women (37).

The prognosis of solid malignancies is widely known to be dependent on the cancer stage, such as the TNM system (38). In this study, BCLC stage was not identified as an independent factor associated with survival. It remains unclear why the stage of HCC was not a risk factor for survival. However, all enrolled patients with HCC were treated with TACE, and patients with BCLC stage B accounted for approximately $80 \%$ of enrolled patients. Therefore, the narrow distribution of HCC stage may be a possible reason that HCC stage was not a risk factor for the prognosis of HCC patients treated with TACE.

Loss of muscle mass is an independent prognostic factor for patients with HCC (11). Therefore, an increase in skeletal muscle mass is thought to be related to the improvement of the prognosis of patients with HCC. We investigated the impact of the change in SMI on survival in patients with HCC; however, an increase in SMI was not identified as an independent prognostic factor in patients with HCC. In addition, there was no significant difference in survival rate between patients with increased SMI and patients with decreased SMI in all patients or in the CR group. Although CR is reported to improve the prognosis of patients with cancer (39), our study indicates 
that $\mathrm{CR}$ may improve the prognosis regardless of changes in muscle mass in patients with HCC.

In this study, we first showed that maintaining physical activity by $\mathrm{CR}$ is more important than increasing muscle mass to improve the prognosis of patients with HCC. The beneficial effect of CR was also observed after propensity score matching. Although it remains unclear why CR improved the prognosis, exercise is reported to exert several beneficial effects against cancer.

First, exercise may suppress the development and proliferation of HCC through modulation of insulin-like growth factor 1 signal. Insulin-like growth factor 1 concentration is related to an increase in tumor growth and development of colon cancer in in vivo and in vitro studies (40). Exercise improves insulin resistance and decreases levels of insulin-like growth factor 1 in patients with breast cancer (39). Moreover, exercise is also known to enhance the expression of p21, insulin-like growth factor-binding protein-3, and PTEN, which suppress insulin-like growth factor 1 signaling in a cancer mouse model (41).

Second, exercise may suppress HCC growth through suppression of the Warburg effect. Aerobic exercise is reported to exert an anti-Warburg effect, leading to an increase in lactate clearance capacity in animal and human studies (42). Lactate is an important metabolic compound involved and necessary in all main sequela for carcinogenesis, specifically: Angiogenesis, immune escape, cell migration, metastasis, and self-sufficient metabolism (42). Lactate metabolism is directly correlated with the prognosis of patients with cancer (43). Thus, exercise-induced alteration in lactate metabolism may suppress HCC growth.

Besides the above mechanisms, myokines are known to suppress cancer cells directly and indirectly (44). Contracting muscle fibers release myokines including interleukin, oncostatin, and secreted protein acidic and rich in cysteine (44). Interleukin is reported to increase mobilization of natural killer lymphocytes and to reduce the growth rate of liver cancer in an in vivo study (45). Oncostatin M was reported to have an anti-proliferative and apoptotic effect on breast-cancer cells in an in vivo study (46). Secreted protein acidic and rich in cysteine is known to be associated with apoptosis of colon cancer cells in mice and humans (47). Thus, a possible hypothesis is that CR may inhibit progression of HCC through up-regulation of myokines, leading to improvement in the prognosis.

There were limitations in this study. This was not a randomized controlled trial with constant duration of CR and irregular time intervals between the first and second CT. Patients who did not agree to exercise were defined as the control group. In addition, the exclusion criteria for the CR group would be associated with death. Thus, patients in the control group might be more deconditioned than patients in the CR group. Second, we did not evaluate the physical activity of patients before TACE and after discharge. Continuous execution of CR should have been evaluated at outpatient visits. Third, changes in eating habits were not evaluated in this study. Since exercise is known to affect eating habits (48) and, changes in eating habits might be a confounding factor of CR. Fourth, there were no patients who underwent liver transplantation during the observation period, suggesting the selection bias. Fifth, we did not evaluate any immune-related variable, although exercise is known to activate immunity and may suppress proliferation of cancer cells through modulation of regulation of the tumor microenvironment $(49,50)$. Thus, multicenter randomized controlled trials should be conducted with constant duration of CR and regular interval of CT examinations, evaluation of physical activity and eating habits before and after TACE, and various treatments for HCC after TACE, including liver transplantation. In addition, effects of CR on immune-related variable should be evaluated to elucidate the mechanisms for CR-related improvement of prognosis in patients with HCC.

In conclusion, we showed that $\mathrm{CR}$ not only increased muscle mass but also prolonged survival in patients with HCC who underwent TACE. Moreover, CR, but not an increase in muscle mass, was an independent factor for survival. Thus, exercise may exert beneficial effects on the prognosis independent from muscle hypertrophy in patients with HCC. Patients with HCC are recommended to maintain physical activity rather than be on bed rest even after a diagnosis of HCC.

\section{Acknowledgements}

The authors would like to thank Ms. Miwa Sakai (Division of Gastroenterology, Department of Medicine, Kurume University School of Medicine) for data acquisition.

\section{Funding}

The present study was supported by Program for Basic and Clinical Research on Hepatitis (AMED; grant no. JP19fk0210045).

\section{Availability of data and materials}

The datasets used and/or analysed during the present study are available from the corresponding author on reasonable request.

\section{Authors' contributions}

$\mathrm{RH}, \mathrm{TK}, \mathrm{SK}$ and KH participated in the study conception and design, data acquisition and interpretation, and manuscript drafting. NG, TY, TO, MB, SI, DN and TN participated in data acquisition and interpretation. AK participated in analysis and interpretation of data. HM, NS and TT participated in data interpretation and revising the manuscript critically for important intellectual content.

\section{Ethics approval and consent to participate}

The study protocol conformed to the ethical guidelines of the Declaration of Helsinki, as reflected in the prior approval given by the Institutional Review Board of Kurume University (approval no. 15072). An opt-out approach was used to obtain informed consent from patients, and personal information was protected during data collection. None of the patients were institutionalized.

\section{Patient consent for publication}

Not applicable. 


\section{Competing interests}

TK has honoraria (lecture fee) from Mitsubishi Tanabe Pharma Corporation, MSD K.K, and Otsuka Pharmaceutical Co., Ltd. The other authors declare that they have no competing interests.

\section{References}

1. Aberg MA, Toren K, Nilsson M, Henriksson M, Kuhn HG, Nyberg J, Rosengren A, Åberg ND and Waern M: Nonpsychotic mental disorders in teenage males and risk of early stroke: A population-based study. Stroke 47: 814-821, 2016.

2. Shimose S, Tanaka M, Iwamoto H, Niizeki T, Shirono T, Aino H, Noda Y, Kamachi N, Okamura S, Nakano M, et al: Prognostic impact of transcatheter arterial chemoembolization (TACE) combined with radiofrequency ablation in patients with unresectable hepatocellular carcinoma: Comparison with TACE alone using decision-tree analysis after propensity score matching Hepatol Res 49: 919-928, 2019.

3. Windsor PM, Nicol KF and Potter J: A randomized, controlled trial of aerobic exercise for treatment-related fatigue in men receiving radical external beam radiotherapy for localized prostate carcinoma. Cancer 101: 550-557, 2004.

4. Serra MC, Ryan AS, Ortmeyer HK, Addison O and Goldberg AP: Resistance training reduces inflammation and fatigue and improves physical function in older breast cancer survivors. Menopause 25: 211-216, 2018.

5. Schmitz KH, Courneya KS, Matthews C, Demark-Wahnefried W, Galvão DA, Pinto BM, Irwin ML, Wolin KY, Segal RJ, Lucia A, et al: American College of Sports Medicine roundtable on exercise guidelines for cancer survivors. Med Sci Sports Exerc 42: 1409-1426, 2010.

6. Mishra SI, Scherer RW, Geigle PM, Berlanstein DR, Topaloglu O, Gotay CC and Snyder C: Exercise interventions on health-related quality of life for cancer survivors. Cochrane Database Syst Rev: CD007566, 2012.

7. Nishikawa H, Shiraki M, Hiramatsu A, Moriya K, Hino K and Nishiguchi S: Japan Society of Hepatology guidelines for sarcopenia in liver disease (1st edition): Recommendation from the working group for creation of sarcopenia assessment criteria. Hepatol Res 46: 951-963, 2016.

8. Tsien C, Davuluri G, Singh D, Allawy A, Ten Have GA, Thapaliya S, Schulze JM, Barnes D, McCullough AJ, Engelen MP, et al: Metabolic and molecular responses to leucine-enriched branched chain amino acid supplementation in the skeletal muscle of alcoholic cirrhosis. Hepatology 61: 2018-2029, 2015.

9. Samoylova ML, Covinsky KE, Haftek M, Kuo S, Roberts JP and Lai JC: Disability in patients with end-stage liver disease: Results from the functional assessment in liver transplantation study. Liver Transpl 23: 292-298, 2017.

10. Koo BK, Kim D, Joo SK, Kim JH, Chang MS, Kim BG, Lee KL and Kim W: Sarcopenia is an independent risk factor for non-alcoholic steatohepatitis and significant fibrosis. J Hepatol 66: 123-131, 2017.

11. Iritani S, Imai K, Takai K, Hanai T, Ideta T, Miyazaki $T$, Suetsugu A, Shiraki M, Shimizu M and Moriwaki H: Skeletal muscle depletion is an independent prognostic factor for hepatocellular carcinoma. J Gastroenterol 50: 323-332, 2015.

12. García-Pagàn JC, Santos C, Barberá JA, Luca A, Roca J, Rodriguez-Roisin R, Bosch J and Rodés J: Physical exercise increases portal pressure in patients with cirrhosis and portal hypertension. Gastroenterology 111: 1300-1306, 1996.

13. Saló J, Guevara M, Fernández-Esparrach G, Bataller R, Ginès A, Jimenez W, Ginès P, Rivera F, Arroyo V and Rodés J: Impairment of renal function during moderate physical exercise in cirrhotic patients with ascites: Relationship with the activity of neurohormonal systems. Hepatology 25: 1338-1342, 1997.

14. Hiraoka A, Michitaka K, Kiguchi D, Izumoto H, Ueki H, Kaneto M, Kitahata S, Aibiki T, Okudaira T, Tomida H, et al: Efficacy of branched-chain amino acid supplementation and walking exercise for preventing sarcopenia in patients with liver cirrhosis. Eur J Gastroenterol Hepatol 29: 1416-1423, 2017.

15. Locklear CT, Golabi P, Gerber L and Younossi ZM: Exercise as an intervention for patients with end-stage liver disease: Systematic review. Medicine (Baltimore) 97: e12774, 2018.
16. Koya S, Kawaguchi T, Hashida R, Goto E, Matsuse H, Saito H, Hirota K, Taira R, Matsushita Y, Imanaga M, et al: Effects of in-hospital exercise on liver function, physical ability, and muscle mass during treatment of hepatoma in patients with chronic liver disease. Hepatol Res 47: E22-E34, 2017.

17. Koya S, Kawaguchi T, Hashida R, Hirota K, Bekki M, Goto E, Yamada M, Sugimoto M, Hayashi S, Goshima N, et al: Effects of in-hospital exercise on sarcopenia in hepatoma patients who underwent transcatheter arterial chemoembolization. J Gastroenterol Hepatol 34: 580-588, 2019.

18. Oken MM, Creech RH, Tormey DC, Horton J, Davis TE, McFadden ET and Carbone PP: Toxicity and response criteria of the Eastern Cooperative Oncology Group. Am J Clin Oncol 5: 649-655, 1982.

19. Citro V, Milan G, Tripodi FS, Gennari A, Sorrentino P, Gallotta G, Postiglione A and Tarantino G: Mental status impairment in patients with West Haven grade zero hepatic encephalopathy: The role of HCV infection. J Gastroenterol 42: 79-82, 2007.

20. Fukui H, Saito H, Ueno Y, Uto H, Obara K, Sakaida I, Shibuya A, Seike M, Nagoshi S, Segawa M, et al: Evidence-based clinical practice guidelines for liver cirrhosis 2015. J Gastroenterol 51: 629-650, 2016.

21. Writing Committee M, Yancy CW, Jessup M, Bozkurt B Butler J, Casey DE Jr, Drazner MH, Fonarow GC, Geraci SA, Horwich T, et al: $2013 \mathrm{ACCF} / \mathrm{AHA}$ guideline for the management of heart failure: A report of the American College of Cardiology Foundation/American Heart Association Task Force on practice guidelines. Circulation 128: e240-e327, 2013.

22. Heart Failure Society of America, Lindenfeld J, Albert NM, Boehmer JP, Collins SP, Ezekowitz JA, Givertz MM, Katz SD, Klapholz M, Moser DK, et al: HFSA 2010 comprehensive heart failure practice guideline. J Card Fail 16: e1-e194, 2010.

23. Rochwerg B, Brochard L, Elliott MW, Hess D, Hill NS, Nava S, Navalesi P Members Of The Steering Committee, Antonelli M, Brozek J, Conti G, et al: Official ERS/ATS clinical practice guidelines: Noninvasive ventilation for acute respiratory failure. Eur Respir J 50, 2017.

24. Wedzicha JA Erc Co-Chair, Miravitlles M, Hurst JR, Calverley PM, Albert RK, Anzueto A, Criner GJ, Papi A, Rabe KF, Rigau D, et al: Management of COPD exacerbations: A European Respiratory Society/American Thoracic Society guideline. Eur Respir J 49, 2017.

25. Saeki I, Yamasaki T, Maeda M, Kawano R, Hisanaga T, Iwamoto T, Matsumoto T, Hidaka I, Ishikawa T, Takami T and Sakaida I: No muscle depletion with high visceral fat as a novel beneficial biomarker of sorafenib for hepatocellular carcinoma. Liver Cancer 7: 359-371, 2018.

26. Garber CE, Blissmer B, Deschenes MR, Franklin BA, Lamonte MJ, Lee IM, Nieman DC and Swain DP; American College of Sports Medicine: American College of Sports Medicine position stand. Quantity and quality of exercise for developing and maintaining cardiorespiratory, musculoskeletal, and neuromotor fitness in apparently healthy adults: Guidance for prescribing exercise. Med Sci Sports Exerc 43: 1334-1359, 2011.

27. Forner A, Reig $M$ and Bruix J: Hepatocellular carcinoma. Lancet 391: 1301-1314, 2018.

28. Kudo M, Matsui O, Izumi N, Iijima H, Kadoya M, Imai Y, Okusaka T, Miyayama S, Tsuchiya K, Ueshima K, et al: JSH consensus-based clinical practice guidelines for the management of hepatocellular carcinoma: 2014 update by the liver cancer study group of Japan. Liver Cancer 3: 458-468, 2014.

29. Teramoto T, Sasaki J, Ishibashi S, Birou S, Daida H, Dohi S, Egusa G, Hiro T, Hirobe K, Iida M, et al: Metabolic syndrome. J Atheroscler Thromb 21: 1-5, 2014.

30. Schneider CA, Rasband WS and Eliceiri KW: NIH Image to Image J: 25 years of image analysis. Nat Methods 9: 671-675, 2012 .

31. Hashida R, Matsuse H, Takano Y, Omoto M, Nago T and Shiba N: Walking exercise combined with neuromuscular electrical stimulation of antagonist resistance improved muscle strength and physical function for elderly people: A pilot study. J Phys Fitness Sports Med 5: 195-203, 2016.

32. Brooks D, Solway S and Gibbons WJ: ATS statement on six-min walk test. Am J Respir Crit Care Med 167: 1287, 2003.

33. Kawaguchi T, Tokushige K, Hyogo H, Aikata H, Nakajima T, Ono M, Kawanaka M, Sawada K, Imajo K, Honda K, et al: A data mining-based prognostic algorithm for NAFLD-related hepatoma patients: A nationwide study by the Japan study group of NAFLD. Sci Rep 8: 10434, 2018. 
34. Dawson JK, Dorff TB, Todd Schroeder E, Lane CJ, Gross ME and Dieli-Conwright CM: Impact of resistance training on body composition and metabolic syndrome variables during androgen deprivation therapy for prostate cancer: A pilot randomized controlled trial. BMC Cancer 18: 368, 2018.

35. Hirota K, Kawaguchi T, Hashida R, Koya S, Bekki M, Goshima N, Yoshiyama T, Otsuka T, Nozoe R, Nakano D, et al: Profiles associated with Sarcopenia in hepatoma patients underwent transcatheter arterial chemoembolization: A data-mining analysis. J Cachexia Sarcopenia Muscle Clin Rep 3: e00066, 2018.

36. Bhasin S, Woodhouse L and Storer TW: Proof of the effect of testosterone on skeletal muscle. J Endocrinol 170: 27-38, 2001.

37. Yakabe $M$, Kojima $T$, Okumura $T$, Takiyama $S$, Umeda-Kameyama Y, Akishita M and Ogawa S: Serum free testosterone levels are positively correlated with skeletal muscle mass in older women aged over 75 years. Geriatr Gerontol Int 19: 460-461, 2019.

38. Okamura Y, Sugiura T, Ito T, Yamamoto Y, Ashida R and Uesaka K: The optimal cut-off value of the preoperative prognostic nutritional index for the survival differs according to the TNM stage in hepatocellular carcinoma. Surg Today 47: 986-993, 2017.

39. Meneses-Echávez JF, Jiménez EG, Rio-Valle JS, Correa-Bautista JE, Izquierdo $\mathrm{M}$ and Ramírez-Vélez R: The insulin-like growth factor system is modulated by exercise in breast cancer survivors: A systematic review and meta-analysis. BMC Cancer 16: 682, 2016.

40. Devin JL, Bolam KA, Jenkins DG and Skinner TL: The influence of exercise on the insulin-like growth factor axis in oncology: Physiological basis, current, and future perspectives. Cancer Epidemiol Biomarkers Prev 25: 239-249, 2016.

41. Yu M, King B, Ewert E, Su X, Mardiyati N, Zhao Z and Wang W: Exercise activates p53 and negatively regulates IGF-1 pathway in epidermis within a skin cancer model.PLoS One 11: e0160939,2016.

42. San-Millán I and Brooks GA: Reexamining cancer metabolism: Lactate production for carcinogenesis could be the purpose and explanation of the Warburg Effect. Carcinogenesis 38: 119-133, 2017.
43. Hofmann P: Cancer and exercise: Warburg hypothesis, tumour metabolism and high-intensity anaerobic exercise. Sports (Basel) 6, 2018.

44. Lucia A and Ramírez M: Muscling in on cancer. N Engl J Med 375: 892-894, 2016.

45. Pedersen L, Idorn M, Olofsson GH, Lauenborg B, Nookaew I, Hansen RH, Johannesen HH, Becker JC, Pedersen KS, Dethlefsen C, et al: Voluntary running suppresses tumor growth through epinephrine- and IL-6-dependent NK cell mobilization and redistribution. Cell Metab 23: 554-562, 2016.

46. Hojman P, Dethlefsen C, Brandt C, Hansen J, Pedersen L and Pedersen BK: Exercise-induced muscle-derived cytokines inhibit mammary cancer cell growth. Am J Physiol Endocrinol Metab 301: E504-E510, 2011

47. Aoi W, Naito Y, Takagi T, Tanimura Y, Takanami Y, Kawai Y, Sakuma K, Hang LP, Mizushima K, Hirai Y, et al: A novel myokine, secreted protein acidic and rich in cysteine (SPARC), suppresses colon tumorigenesis via regular exercise. Gut 62: 882-889, 2013.

48. Panão I and Carraça EV: Effects of exercise motivations on body image and eating habits/behaviours: A systematic review. Nutr Diet, 2019.

49. Kerr J, Anderson C and Lippman SM: Physical activity, sedentary behaviour, diet, and cancer: An update and emerging new evidence. Lancet Oncol 18: e457-e471, 2017.

50. Koelwyn GJ, Quail DF, Zhang X, White RM and Jones LW: Exercise-dependent regulation of the tumour microenvironment. Nat Rev Cancer 17: 620-632, 2017.

This work is licensed under a Creative Commons Attribution-NonCommercial-NoDerivatives 4.0 International (CC BY-NC-ND 4.0) License. 